\title{
LEVANTAMENTO ETNOBOTÂNICO DE ESPÉCIES VEGETAIS UTILIZADAS NA ODONTOLOGIA NO RECÔNCAVO BAIANO
}

\author{
ETHNOBOTANICAL SURVEY OF PLANT SPECIES USED IN DENTISTRY IN THE \\ RECÔNCAVO BAIANO, BRAZIL
}

LEVANTAMIENTO ETNOBOTÁNICO DE ESPECIES VEGETALES UTILIZADAS EN ODONTOLOGÍA EN EL RECÔNCAVO BAIANO, BRAZIL

Geovane Silva de Araújo 1

Elba Brito dos Santos 2

Patrícia Pinto dos Santos Silva ${ }^{3}$

Vania Jesus dos Santos de Oliveira ${ }^{4}$

Noelma Miranda de Brito ${ }^{5}$

Palavras-chave: Odontologia Comunitária; Fitoterapia; Plantas Medicinais.

Keywords: Community Dentistry; Phytotherapy; Plants, Medicinal.

Palabras clave: Odontología Comunitaria; Fitoterapia; Plantas Medicinales.

Submetido: $28 / 06 / 2017$

Aprovado: $24 / 11 / 2017$

Autor(a) para Correspondência: Geovane Silva de Araújo End: Rua 05, Papagaio, Feira de Santana

CEP: 44059-680, Bahia E-mail:geovane.ba@outlook.com

\section{RESUMO}

O emprego de plantas medicinais para tratar enfermidades é uma prática milenar. Nas comunidades tradicionais brasileiras, as plantas medicinais são adotadas em forma de chás e fitoterápicos no tratamento de diversas enfermidades - dentre elas as patologias bucais. Este artigo apresenta um levantamento etnobotânico de espécies vegetais comercializadas para tratar patologias bucais em Cruz das Almas, município do Recôncavo Baiano. Aplicou-se um questionário sobre a adoção de plantas medicinais na saúde bucal aos raizeiros e aos usuários na sala de espera de unidades da Estratégia Saúde da Família (ESF) de Cruz das Almas. As plantas mais comercializadas pelos raizeiros foram: sara-tudo (Byrsonima intermedia A. Juss.); fruto da noz-moscada (Myristica fragrans Houtt.); aroeira (Schinus terebinthifolius Raddi); dente-de-cravo (Syzygium aromaticum L. Merrill \& Perry); e misturas, como as 7 ervas. Já os usuários da ESF dizem recorrer a sara-tudo (B. intermedia) para alívio de dor de dente e inflamação. Evidências disponiveis na literatura científica comprovam a eficácia de tais plantas no tratamento de patologias bucais. Contudo, mostra-se necessário investigar em maior profundidade a ação dessas plantas medicinais.

1. Estudante de Graduação em Farmácia na Faculdade Maria Milza (Famam). Governador Mangabeira (BA), Brasil. E-mail: geovannedearaujo@gmail.com

2. Estudante de Graduação em Farmácia na Famam. Governador Mangabeira (BA),Brasil. E-mail: elbabritods@ hotmail.com

3. Estudante de Graduação em Farmácia na Famam. Governador Mangabeira (BA),Brasil. E-mail: 01patyps@ gmail.com

4. Agrônoma. Doutora em Farmácia. Professora na Famam. Governador Mangabeira (BA), Brasil. E-mail: vania79br@yahoo.com.br

5. Engenheira agrônoma. Doutora em Ciências Agrárias. Professora na Famam. Governador Mangabeira (BA), Brasil. E-mail: britonoelma@yahoo.com.br 


\section{ABSTRACT}

The use of medicinal plants to treat diseases is an age-old practice. In the traditional Brazilian communities, medicinal plants have been adopted as teas and herbal medicines in the treatment of various diseases - among them oral pathologies. This article presents an ethnobotanical survey of plant species commercialized to treat oral pathologies in Cruz das Almas, a municipality in the Recôncavo Baiano, Brazil. A questionnaire on the adoption of medicinal plants in oral health was applied to root workers and users in the waiting room of Family Health Strategy (FHS) units in Cruz das Almas. The plants most widely commercialized by root workers were: 'sara-tudo' (Byrsonima intermedia A. Juss.); nutmeg fruit (Myristica fragrans Houtt.); Brazilian peppertree (Schinus terebinthifolius Raddi); clove (Syzygium aromaticum L. Merrill \& Perry); and mixtures, like the 7 herbs. FHS users are said to resort to 'sara-tudo' (B. intermedia) for relief of tooth pain and inflammation. Evidence available in the scientific literature confirms the efficacy of such plants in the treatment of oral pathologies. However, there is a need to investigate in greater depth the action of these medicinal plants.

\section{RESUMEN}

El empleo de plantas medicinales para tratar enfermedades es una práctica milenaria. En comunidades tradicionales brasileñas, las plantas medicinales se adoptan en forma de tés y fitoterápicos en el tratamiento de diversas enfermedades - entre ellas las patologías bucales. Este artículo presenta un levantamiento etnobotánico de especies vegetales comercializadas para tratar patologías bucales en Cruz das Almas, municipio del Recôncavo Baiano. Se aplicó un cuestionario sobre la adopción de plantas medicinales en la salud bucal a los raiceros y a los usuarios en la sala de espera de unidades de la Estrategia Salud de la Familia (ESF) en Cruz das Almas. Las plantas más comercializadas por los raiceros fueron: 'sara-tudo' (Byrsonima intermedia A. Juss.); fruto de nuez moscada (Myristica fragrans Houtt.); aroeira (Schinus terebinthifolius Raddi); árbol del clavo (Syzygium aromaticum L. Merrill \& Perry); y mezclas, como las 7 hierbas. A su vez, los usuarios da la ESF dicen recurrir a 'sara-tudo' (B. intermedia) para aliviar el dolor de diente y la inflamación. Evidencias disponibles en la literatura científica demuestran la eficacia de tales plantas en el tratamiento de patologías bucales. Sin embargo, es necesario investigar en mayor profundidad la acción de estas plantas medicinales.

\section{INTRODUÇÃO}

Desde os primórdios há relatos da utilização de plantas medicinais com o intuito de tratar enfermidades. Com base nisso, por meio do conhecimento acerca das propriedades medicinais de plantas acumulado ao longo de gerações, o homem pode utilizá-las na terapia convencional. Nas comunidades tradicionais brasileiras, as plantas medicinais são adotadas como chás e fitoterápicos ${ }^{1}$; em muitas dessas comunidades, o principal mecanismo de renda é a agricultura e predomina o cultivo próprio de plantas medicinais para uso como remédios caseiros $^{2}$.

A manifestação cultural do uso de plantas está diretamente relacionada à etnobotânica, que inclui o modo, os costumes e as tradições das populações e sua interação com a natureza ${ }^{3}$. A etnobotânica está diretamente ligada à fitoterapia - definida como ramo das Ciências Médicas que utiliza plantas medicinais e drogas vegetais para tratar enfermidades sem recorrer a substâncias de outra origem ${ }^{4}$.

Do ponto de vista sociocultural, a fitoterapia se mostra muito importante, uma vez que na maioria das vezes os usuários dos serviços de saúde veem as plantas medicinais como meras opções terapêuticas paliativas ${ }^{5}$; porém, na perspectiva dos profissionais de saúde, o desconhecimento acerca das indicações e dos cuidados no uso de plantas medicinais ainda constitui um problema ${ }^{6}$.

Apesar das inúmeras possibilidades de uso de plantas medicinais por parte dos profissionais da saúde, o potencial das plantas medicinais tem sido pouco explorado na odontologia, seja para tratar doenças bucais ou doenças sistêmicas com manifestações bucais ${ }^{6}$. 0 maior avanço nesse sentido se refere às plantas com ação antimicrobiana e antifúngica, devido ao considerável avanço no desenvolvimento de derivados naturais com propriedades bactericidas ou fungicidas ${ }^{7}$.

Assim, com base nessas informações, este artigo apresenta um levantamento etnobotânico de espécies vegetais comercializadas para tratar patologias bucais em Cruz das Almas, município do Recôncavo Baiano. 


\section{METODOLOGIA}

A pesquisa foi realizada em 5 unidades da Estratégia Saúde da Família (ESF) e na feira livre do centro de Cruz das Almas - onde se investigou o uso tradicional de plantas medicinais com vistas à saúde bucal.

0 levantamento etnobotânico foi realizado com 2 etapas de entrevistas: a) dos 10 raizeiros que comercializavam plantas medicinais na feira livre; e b) dos 31 usuários presentes na sala de espera das unidades da ESF. Os raizeiros responderam questões acerca de seus produtos, que correspondem às informações repassadas para a população a respeito das plantas medicinais que serão consumidas. Já os usuários da ESF responderam questões sobre 0 consumo dessas plantas medicinais como remédios e sua efetividade terapêutica.

Os dados obtidos foram tabulados e analisados por meio das técnicas de estatística descritiva, com apresentação de percentuais de frequência.

Este estudo seguiu os preceitos da Resolução n. 466/2012, do Conselho Nacional de Saúde (CNS), que dispõe sobre a pesquisa envolvendo seres humanos, e foi aprovado pelo Comitê de Ética em Pesquisa da
Universidade Federal do Recôncavo da Bahia (UFRB), sob o Parecer n. 1.063.134/2015.

\section{RESULTADOS}

Foram entrevistados 10 raizeiros ( 6 homens e 4 mulheres): 2 com 40 a 49 anos de idade, 5 com 50 a 59 anos e 3 com 60 a 69 anos. Esses comerciantes podem ser apontados como representantes de gerações mais antigas, tendo em vista que a média foi de 57,6 anos de idade entre os homens e de 51 anos entre as mulheres (com variação de 46 a 68 anos).

Em relação à escolaridade, houve prevalência do Ensino Fundamental (7 dos 10 raizeiros). Parte dos entrevistados relatou ter enfrentado dificuldades para estudar, pois a maioria era de origem rural e teria de locomover-se para a zona urbana mais próxima para ter acesso a uma escola.

Dos 31 usuários da ESF entrevistados, $21(67,7 \%)$ são mulheres. A média de idade foi de 41 anos entre as mulheres (variação de 21 a 67 anos) e de 45 anos entre os homens (variação de 23 a 63 anos), como ilustra a Tabela 1.

Tabela 1 - Caracterização dos usuários da ESF entrevistados no levantamento etnobotânico. Cruz das Almas, 2015.

\begin{tabular}{|c|c|c|c|c|}
\hline \multirow{3}{*}{ Faixa etária } & \multicolumn{4}{|c|}{ Sexo } \\
\hline & \multicolumn{2}{|c|}{ Masculino } & \multicolumn{2}{|c|}{ Feminino } \\
\hline & $(\%)$ & $\mathrm{N}$ & $(\%)$ & $\mathrm{N}$ \\
\hline 20-29 anos & 10 & 3 & 19 & 6 \\
\hline 30-39 anos & 3 & 1 & 13 & 4 \\
\hline 40-49 anos & 3 & 1 & 6,5 & 2 \\
\hline 50-59 anos & 13 & 4 & 6,5 & 2 \\
\hline $60-69$ anos & 13 & 4 & 13 & 4 \\
\hline Total & 42 & 13 & 58 & 18 \\
\hline
\end{tabular}

Fonte: Elaborada pelos autores.

Dentre os entrevistados, $9(29 \%)$ disseram não ser alfabetizados ou não ter estudo formal e $2(6 \%)$ tinham completo (Tabela 2). Todos vivem em área urbana, porém, uma parte significativa relata ser de origem rural e apresenta grande influência dos costumes e das práticas de seus avós e demais parentes de origem rural.

Tabela 2 - Escolaridade dos usuários da ESF entrevistados no levantamento etnobotânico. Cruz das Almas, 2015.

\begin{tabular}{lcc}
\hline Escolaridade & $(\%)$ & $\mathbf{N}$ \\
\hline Sem estudo formal & 29 & 9 \\
Ensino Fundamental incompleto & 16 & 5 \\
Ensino Fundamental completo & 26 & 8 \\
Ensino Médio incompleto & 10 & 3 \\
Ensino Médio completo & 13 & 4 \\
Ensino Superior completo & 6 & 2 \\
\hline Total & 100 & 31 \\
\hline
\end{tabular}

Fonte: Elaborada pelos autores. 
Os resultados demonstraram que todos os raizeiros da feira livre do centro de Cruz das Almas comercializam plantas medicinais para tratar patologias bucais. Dentre as plantas utilizadas, 4 raizeiros indicaram que a mais vendida é a sara-tudo (Byrsonima intermedia A. Juss.). 0 fruto da nozmoscada (Myristica fragrans Houtt.), a aroeira (Schinus terebinthifolius Raddi) e o dente-de-cravo (Syzygium aromaticum L. Merrill \& Perry) também são plantas medicinais bastante comercializadas (relatos de 3 entrevistados). Misturas, como as 7 ervas - isto é, manjericão (Ocimum basilicum L.), alecrim (Rosmarinus officinalis L.), espada-de-são-jorge (Sansevieria trifasciata Prain, 1903), comigoninguém-pode (Dieffenbachia amoena Hort. ex L. Gentil), pimenta-do-reino (Capsicum spp.), guiné (Petiveria alliacea L.) e arruda (Ruta graveolen L.) são vendidas por 2 raizeiros e as pessoas acreditam que seu chá é mais potente e eficaz devido à combinação de espécies vegetais. Outras plantas, como tansagem (Plantago major L.) e erva-cidreira (Melissa officinalis L.), são comercializadas por 1 raizeiro.

Dentre as complicações dentárias relatadas pelos raizeiros como mais comuns entre os usuários se destacam a dor de dente e a gengivite - a planta mais indicada nesses casos é a tansagem ( $P$. major), que os comerciantes dizem conter propriedades antiinflamatórias. Sua forma de uso geralmente é tópica em casos de inflamação (Tabela 3). Entretanto, há predomínio da venda da casca de sara-tudo ( $B$. intermedia); de acordo com os raizeiros, seu chá tem potencial efeito analgésico e combate a inflamação, por isso é indicada para a dor de dente. Além dessas plantas, o alecrim ( $R$.officinalis), a erva-cidreira ( $M$. officinalis) e a tansagem ( $P$. major) são compradas pelos usuários para tratar úlceras bucais. 0s comerciantes acreditam que essas plantas têm propriedades antibacterianas e anti-inflamatórias.

Tabela 3 - Plantas medicinais comercializadas em feira livre para tratar patologias bucais. Cruz das Almas, 2015.

\begin{tabular}{|c|c|c|c|c|}
\hline Táxons & NP & PU & FP & FU \\
\hline B. intermedia & Sara-tudo & Casca do caule seca & Decocção & Tomar 2 vezes ao dia \\
\hline M. fragrans & Noz-moscada & $\begin{array}{l}\text { Semente seca e } \\
\text { triturada }\end{array}$ & Infusão & Tomar 1 vez ao dia \\
\hline S. terebinthifolius & Aroeira & Casca do caule seca & Decocção & Gargarejo 2 vezes ao dia \\
\hline 0. basilicum & Manjericão & Folhas e caules & Infusão & Enxague bucal e gargarejo \\
\hline R. officinalis & Alecrim & Folhas & Infusão & Tomar 2 vezes ao dia \\
\hline Capsicum spp. & $\begin{array}{l}\text { Pimenta-do- } \\
\text { reino }\end{array}$ & Fruto & Extrato & $\begin{array}{c}\text { Diluído em água para } \\
\text { enxague bucal e aplicação } \\
\text { tópica no dente }\end{array}$ \\
\hline S. aromaticum & Dente-de-cravo & Botão da flor seco & $\begin{array}{l}\text { Extrato ou } \\
\text { decocção }\end{array}$ & $\begin{array}{c}\text { Enxague bucal e aplicação } \\
\text { tópica no dente }\end{array}$ \\
\hline P. alliacea & Guiné & Folha & Infusão & $\begin{array}{c}\text { Gargarejo de } 3 \text { a } 4 \text { vezes ao } \\
\text { dia }\end{array}$ \\
\hline P. major & Tansagem & Folha & Infusão & $\begin{array}{c}\text { Gargarejo e aplicação } \\
\text { tópica no dente e na } \\
\text { gengiva }\end{array}$ \\
\hline M. officinalis & Erva-cidreira & Folhas & Infusão & Tomar 2 vezes ao dia \\
\hline
\end{tabular}

Legenda: NP = nome popular; PU = parte utilizada, FP = forma de preparo; FU = forma de uso.

Fonte: Elaborada pelos autores.

Os raizeiros informam, ainda, que o óleo essencial do cravo-da-índia (S. aromaticum) e da pimenta-doreino (Capsicum spp.) é predominantemente comercializado em forma de extrato ou óleo essencial e é aplicando pelos usuários no dente para aliviar a dor.

Dentre os usuários atendidos nas unidades da ESF, $24(76 \%)$ utilizam plantas medicinais para tratar patologias. As plantas medicinais são utilizadas para fins odontológicos por 18 indivíduos (57\%), sendo que 12 (39\%) adotam sara-tudo (B. intermedia), 9
(28\%) recorrem a erva-cidreira (M. officinalis), 6 $(18 \%)$ empregam tansagem ( $P$. major) e $3(9 \%)$ usam mastruz (Chenopodium ambrosioides L.).

De acordo com o levantamento deste estudo, saratudo ( $B$. intermedia) é a planta mais adotada para tratamento odontológico pelos usuários da ESF: 12 entrevistados $(39 \%)$. Esse achado coincide com os relatos da planta mais comercializada por raizeiros. A planta mastruz (C. ambrosioides), pouco citada pelos raizeiros, foi bastante citada pelos usuários da ESF estes relatam tanto utilizá-la como cultivá-la no 
quintal de casa: 14 (44\%) adquiriram o hábito da fitoterapia por meio de seus avós, que detinham bastante conhecimento acerca dessa prática.

Dentre as plantas com relato de efeito adverso, destaca-se a erva-cidreira (M. officinalis), que além de analgésica tem potencial calmante, por isso, muitos usuários da ESF dizem apresentar diminuição de sua pressão arterial após a ingestão do chá dessa planta para aliviar a dor de dente. Todos os usuários da ESF dizem que os medicamentos são mais efetivos do que as plantas por apresentar efeito imediato no tratamento, porém, alguns afirmam que as plantas também são bastante eficazes. A maioria dos usuários da ESF não informou ao odontologista que recorre a planta medicinal.

\section{DISCUSSÃO}

$\mathrm{Na}$ literatura, há ensaios clínicos com plantas medicinais que comprovam cientificamente sua ação na terapia bucal. A maioria dos ensaios in vitro indicam atividade antibacteriana e os ensaios in vivo indicam atividade anti-inflamatória (Tabela 4). Já no levantamento deste estudo, a maioria dos usuários relata utilizar tais plantas para fins de antissepsia bucal e inflamações.

Tabela 4 - Ensaios clínicos com plantas medicinais que abordam seu potencial terapêutico.

\begin{tabular}{|c|c|c|c|}
\hline Planta & Ensaios & Forma fitoterápica & Referência \\
\hline \multirow{3}{*}{ B. intermedia } & $\begin{array}{l}\text { Atividade anti-inflamatória (in } \\
\text { vivo) }\end{array}$ & Extrato da folha & Moreira et al., $2011^{10}$ \\
\hline & $\begin{array}{c}\text { Atividade antimicrobiana (in } \\
\text { vitro) }\end{array}$ & Extrato da folha & Moraes $2006^{9}$ \\
\hline & $\begin{array}{c}\text { Atividade anti-inflamatória (in } \\
\text { vivo) }\end{array}$ & Extrato da casca do caule & Orlandi et al., $2011^{11}$ \\
\hline \multirow{3}{*}{ M. fragrans } & Atividade antibiofilme (in vitro) & Extrato da semente & Yanti et al., $2008^{12}$ \\
\hline & $\begin{array}{c}\text { Atividade antimicrobiana (in } \\
\text { vitro) }\end{array}$ & Extrato da semente & Chung et al., $2006^{13}$ \\
\hline & $\begin{array}{l}\text { Isolamento de inibidores da } \\
\text { produção de óxido nítrico }\end{array}$ & Extrato da semente & Cao et al., $2013^{14}$ \\
\hline \multirow{3}{*}{ S. terebinthifolius } & $\begin{array}{c}\text { Reduções dos índices de placa e } \\
\text { de sangramento gengival }\end{array}$ & Extrato da folha & Lins et al., $2013^{15}$ \\
\hline & $\begin{array}{l}\text { Atividade anti-inflamatória e } \\
\text { cicatrizante (in vivo) }\end{array}$ & Extrato da folha & Martorelli al., $2011^{16}$ \\
\hline & Atividade antifúngica & $\begin{array}{c}\text { Tintura da casca do caule } \\
\text { seca }\end{array}$ & Freires et al., $2011^{17}$ \\
\hline \multirow{3}{*}{ S. aromaticum } & $\begin{array}{c}\text { Redução do crescimento micelial } \\
\text { (in vitro) }\end{array}$ & $\begin{array}{c}\text { Óleo essencial dos botões } \\
\text { florais }\end{array}$ & Santos et al., $2007^{20}$ \\
\hline & $\begin{array}{c}\text { Atividade antimicrobiana (in } \\
\text { vitro) }\end{array}$ & $\begin{array}{c}\text { Óleo essencial dos botões } \\
\text { florais }\end{array}$ & $\begin{array}{l}\text { Rodríguez et al., } \\
\qquad 2014^{18}\end{array}$ \\
\hline & $\begin{array}{l}\text { Atividade anti-inflamatória e } \\
\text { cicatrizante (in vivo) }\end{array}$ & Extrato dos botões florais & Valente $2006^{19}$ \\
\hline P. alliacea & $\begin{array}{c}\text { Atividade de antimicrobiana (in } \\
\text { vitro) }\end{array}$ & $\begin{array}{c}\text { Extratos brutos (partes } \\
\text { aéreas) }\end{array}$ & Guedes et al., $2009^{21}$ \\
\hline \multirow{2}{*}{ M. officinalis } & $\begin{array}{c}\text { Atividade anti-inflamatória (in } \\
\text { vivo) }\end{array}$ & Extrato da folha & Bounihi et al., $2013^{24}$ \\
\hline & $\begin{array}{c}\text { Identificação de compostos } \\
\text { antibacterianos }\end{array}$ & óleo essencial & $\begin{array}{l}\text { H ncianu et al., } \\
2008^{22}\end{array}$ \\
\hline
\end{tabular}

Fonte: Elaborada pelos autores.

Em relação ao costume de utilizar plantas medicinais, os resultados deste estudo se assemelham a outras publicações ${ }^{8}$, pois os usuários relatam que vem dos avós a influência para o uso de plantas medicinais, decorrente de seu maior conhecimento sobre a "cura" por meio de plantas.

$\mathrm{Na}$ literatura, a $B$. intermedia é predominantemente citada em ensaios clínicos in vivo e in vitro. Outro estudo 
comprova inibição in vitro do crescimento de cepas de Escherichia coli, Staphylococcus aureus, Yersinia enterocolitica e Aeromonas hydrophila, por meio do extrato da folha da $B$. intermedia ${ }^{9}$. E existem relatos da atividade anti-inflamatória do extrato da casca e da folha de $B$. intermedia, em ensaios in vivo realizados com ratos ${ }^{10,11}$.

Por meio do isolamento da macelignan da semente de $M$. fragrans, detectou-se atividade contra colonizadores primários orais; os resultados do ensaio clínico com $M$. fragrans comprovam a atividade antibacteriana contra a Streptococcus mutans ${ }^{12}$. Também foram identificados 6 eolignanas e dihidrobenzofuranos, compostos inibidores da produção de óxido nítrico ${ }^{13,14}$.

Existem evidências, proporcionadas por estudos clínicos, da significativa redução dos índices de placa e de sangramento gengival por meio do gargarejo do extrato hidroalcoólico da folha da S. terebinthifolius ${ }^{15}$. Em outros ensaios in vivo, o extrato hidroalcoólico da folha de $S$. terebinthifolius apresenta atividade antiinflamatória em ratos ${ }^{16}$. No ensaio in vitro de Freires et al. ${ }^{17}$, a tintura de sua casca apresenta atividade antifúngica diante das cepas de Candida spp.

Em ensaios utilizando o óleo essencial de botões florais secos de $S$. aromaticum há testes de inibição microbiana de ensaio in vitro, inibindo Streptococcus mutans ${ }^{18}$, e inflamatória em ensaios in vivo ${ }^{19}$. Além disso, segundo relato da literatura, o óleo essencial da $S$. aromaticum reduz o crescimento micelial in vitro de Fusarium oxysporum e Rhizoctonia solani ${ }^{20}$.

Relatos da literatura comprovam que o extrato bruto da $P$. alliacea apresenta potencial antibacteriano diante das bactérias Bacilus subtilis, Pseudomonas aeruginosa, Escherichia coli, Streptococcus mutans, Staphylococcus aureus, Staphylococcus epidermidis, Enterococcus faecalis ${ }^{21}$.

A M. officinalis, além de propriedades relaxantes, apresenta ensaios confirmando na literatura que 0 extrato tem potencial anti-inflamatório. Um estudo fotoquímico identificou compostos com potencial antibacteriano citral (neral e geranial), citronelal e transcariofileno no óleo essencial da M. officinalis ${ }^{22}$.

\section{CONCLUSÃO}

Esta pesquisa possibilitou a constatação de que a faixa etária dos raizeiros não variou muito e é alta, visto que decorre da cultura de gerações mais antigas de feirantes. Entretanto, a busca dessas plantas pelos usuários ainda se mostra ascendente, mesmo no que

\section{0 uso de plantas medicinais como prática terapêutica pela população é uma prática constante.}

diz respeito à saúde bucal - há usuários de ambos os sexos e de todas as idades.

Esta pesquisa confirma, ainda, que a transmissão de conhecimentos acerca de plantas medicinais advém de gerações mais antigas, tendo em vista que a maioria dos usuários afirmou adquirir informações sobre as plantas medicinais com os parentes mais velhos, como os avós. 0s raizeiros participantes desta pesquisa comercializam diversas plantas medicinais voltadas a tratamento odontológico.

0 cultivo dessas plantas pelos feirantes de Cruz das Almas ocorre na modalidade orgânica, predominantemente comercializada como droga vegetal, porém, tais plantas também são mantidas in natura. Cruz das Almas é um município com bom mercado consumidor, inclusive para tratamento odontológico, uma vez que grande parte dos usuários da ESF afirmou ter obtido a planta no comércio.

Segundo os resultados levantados em ensaios clínicos utilizando as plantas mais adotadas pelos usuários, observou-se que as plantas mais comuns, como sara-tudo ( $B$. intermedia), noz-moscada ( $M$. fragrans), aroeira ( $S$. terebinthifolius) e dente-decravo (S. aromaticum), apresentam comprovação científica de atividade antibacteriana, prevenindo e combatendo temporariamente o surgimento de cáries, além da dor e das inflamações.

0 uso de plantas medicinais como prática terapêutica pela população é uma prática constante. Isso possibilita que se estabeleça uma troca entre o conhecimento empírico e o conhecimento científico - relacionados às práticas das regiões em questão nos estudos científicos ${ }^{23}$.

\section{CONTRIBUIÇÃO DOS AUTORES}

Geovane Silva de Araújo contribuiu com a concepção e realização da pesquisa e a estruturação e redação do manuscrito. Elba Brito dos Santos e Patrícia Pinto dos Santos Silva contribuíram com a estruturação e redação do manuscrito. Vania Jesus dos Santos de Oliveira e Noelma Miranda de Brito 
contribuíram com a redação e revisão crítica do manuscrito.

\section{REFERÊNCIAS}

1. Vieira SCH, Sólon S, Vieira MC, Zárate NAH. Levantamento de fitoterápicos manipulados em farmácias magistrais de Dourados-MS. Rev Bras Farmacogn [serial on the internet]. 2010 [cited 2018 Apr 22];20(1):28-34. Available from: http://www.scielo.br/pdf/rbfar/v20n1/v20n1a07.pdf

2. Oliveira FCS, Barros RFM, Moita Neto JM. Plantas medicinais utilizadas em comunidades rurais de 0eiras, semiárido piauiense. Rev Bras Plantas Med [serial on the internet]. 2010 [cited 2018 Apr 22];12(3):282-301. Available from: http://www.scielo.br/pdf/rbpm/v12n3/06. pdf

3. Albuquerque UP, Lucena RFP, Cunha LVFC. Métodos e técnicas na pesquisa etnobotânica. 2. ed. Recife: Comunigraf; 2008.

4. Carvalho JCT. Fitoterápicos anti-inflamatórios: aspectos químicos, farmacológicos e aplicações terapêuticas. Ribeirão Preto (SP): Tecmedd; 2004.

5. Ness J, Sherman FT, Pan CX. Alternative medicine: what the data say about common herbal therapies. Geriatrics. 1999;54(10):33-8.

6. Oliveira CJ, Araujo CJ. Plantas medicinais: usos e crenças de idosos portadores de hipertensão arterial. Rev Eletrônica Enferm [serial on the internet]. 2007 [cited 2018 Apr 22];9(1):93-105. Available from: https://www.fen.ufg. br/revista/v9/n1/pdf/v9n1a07.pdf

7. Das K, Tiwari RKS, Shrivastava DK. Techniques for evaluation of medicinal plant products as antimicrobial agents: current methods and future trends. Journal of Medicinal Plants Research [serial on the internet]. 2010 [cited 2018 Apr 22];4(2):104-11. Available from: http:// www.academicjournals.org/article/article 1380375549 Das\%20et\%20al.pdf

8. Borba AM, Macedo M. Plantas medicinais usadas para a saúde bucal pela comunidade do bairro Santa Cruz, Chapada dos Guimarães, MT, Brasil. Acta Bot Bras [serial on the internet]. 2006 [cited 2018 Apr 22];20(4):771-82. Available from: http://www.scielo.br/pdf/abb/v20n4/03.pdf

9. Moraes HP. Avaliação in vitro da atividade antibacteriana de extratos de Byrsonima spp. e Alchornea spp.: estudo comparativo entre as técnicas de diluição em tubos e microplacas. Araraquara (SP): Unesp; 2006.

10. Moreira LQ, Vilela FC, Orlandi L, Dias DF, Santos AL, Da Silva MA, et al. Anti-inflammatory effect of extract and fractions from the leaves of Byrsonima intermedia A. Juss. in rats. J Ethnopharmacol [serial on the internet]. 2011 [cited 2018 Apr 22];138:610-5. Available from: https://www. sciencedirect.com/science/article/pii/S0378874111007331
11. Orlandi L, Vilela FC, Santa-Cecília FV, Dias DF, Alves-DaSilva G, Giusti-Paiva A. Anti-inflammatory and antinociceptive effects of the stem bark of Byrsonima intermedia A. Juss. J Ethnopharmacol [serial on the internet]. 2011 [cited 2018 Apr 22];137(3):1469-76. Available from: https://www.sciencedirect.com/science/ article/pii/S0378874111006052

12. Yanti, Rukayadi Y, Kim KH, Hwang JK. In vitro antibiofilm activity of macelignan isolated from Myristica fragrans Houtt. against oral primary colonizer bacteria. Phytother Res. 2008;22(3):308-12.

13. Chung JY, Choo JH, Lee MH, Hwang JK. Anticariogenic activity of macelignan isolated from Myristica fragrans (nutmeg) against Streptococcus mutans. Phytomedicine. $2006 ; 13(4): 261-6$.

14. Cao GY, Yang XW, Xu W, Li F. New inhibitors of nitric oxide production from the seeds of Myristica fragrans. Food Chem Toxicol. 2013;62:167-71.

15. Lins R, Vasconcelos FHP, Leite RB, Coelho-Soares RS, Barbosa DN. Avaliação clínica de bochechos com extratos de Aroeira (Schinus terebinthifolius) e Camomila (Matricaria recutita L.) sobre a placa bacteriana e a gengivite. Rev Bras Plantas Med [serial on the internet]. 2013 [cited 2018 Apr 22];15(1):112-20. Available from: http://www.scielo.br/ $\mathrm{pdf} / \mathrm{rbpm} / \mathrm{v} 15 \mathrm{n} 1 / \mathrm{a} 16 \mathrm{v} 15 \mathrm{n} 1 . \mathrm{pdf}$

16. Martorelli SBF, Pinheiro AL, Souza IA, Higino JS, Bravo F. Efeito anti-inflamatório e cicatrizante do extrato hidroalcoólico de Schinus terebinthifolius Raddi (Aroeira) a $30 \%$ em orabase - estudo "in vivo". Int J Dent. 2011;10(2):80-90.

17. Freires IA, Alves LA, Jovito VC, Castro RD. Atividade antifúngica de Schinus terebinthifolius (Aroeira) sobre cepas do gênero Candida. Revista Odontológica do Brasil-Central [serial on the internet]. 2011 [cited 2018 Apr 22];20(52):415. Available from: http://robrac.org.br/seer/index.php/ ROBRAC/article/view/491/534

18. Rodríguez $0 E$, Sánchez $R$, Verde $M$, Núnez $M$, Ríos $R$, Chávez A. Obtaining of the essential oil of Syzygium aromaticum, identification of eugenol and its effect on Streptococcus mutans. J Oral Res [serial on the internet]. 2014 [cited 2018 Apr 22];3(4):218-24. Available from: http://www.joralres.com/index.php/J0R/article/ view/104/120

19. Valente ROH. Avaliação das propriedades tóxicas, antiinflamatórias e cicatrizantes do extrato de cravo-da-índia Syzygium aromaticum (L) Merr. e LM Perry. João Pessoa: Universidade Federal da Paraíba; 2006.

20. Santos LGM, Cardoso MG, Lima RK, Souza PE, Guimarães LGL, Andrade MA. Avaliação do potencial fungitóxico do óleo essencial de Syzygium aromaticum (L.) Merr \& Perry (Cravo-da-Índia). Tecno-Lógica [serial on the internet]. 2007 [cited 2018 Apr 22];11(1):11-4. Available from: https://online.unisc.br/seer/index.php/tecnologica/ article/view/154/125 
21. Guedes RCM, Nogueira NGP, Fusco Almeida AM, Souza CRF, Oliveira WP. Atividade antimicrobiana de extratos brutos de Petiveria alliacea L. Latin American Journal of Pharmacy [serial on the internet]. 2009 [cited $2018 \mathrm{Apr}$ 22];28(4):520-4. Available from: http://www.latamjpharm. org/trabajos/28/4/LAJ0P_28_4_1_7_AIT6W1N9TT.pdf

22. Hancianu M, Aprotosoaie AC, Gille E, Poiat A, Tuchilu $C$, Spac $A$, et al. Chemical composition and in vitro antimicrobial activity of essential oil of Melissa officials L. from Romania. Rev Med Chir Soc Med Nat Iasi. $2008 ; 112(3): 843-7$.

23. Teixeira AH, Bezerra MM, Chaves HV, Val DR, Pereira Filho S, Rodrigues e Silva MAA. Conhecimento popular sobre o uso de plantas medicinais no município de Sobral-Ceará, Brasil. Sanare (Sobral, Online) [serial on the internet]. 2014 [cited 2018 Apr 22];12(1):23-8. Available from: https://sanare.emnuvens.com.br/sanare/article/ view $/ 429 / 284$

24. Bounihi A, Hajjaj G, Alnamer R, Cherrah Y, Zellou A. In Vivo Potential Anti-Inflammatory Activity of Melissa officinalis L. Essential 0il. Adv Pharmacol Sci. 2013; 2013(1): 1-7.

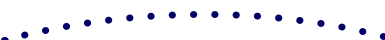

$\ldots \ldots \ldots \ldots \ldots$

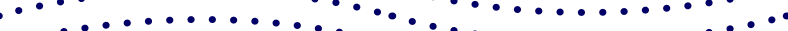
$\cdots \cdots$ $\cdots \cdots \cdots \cdots$
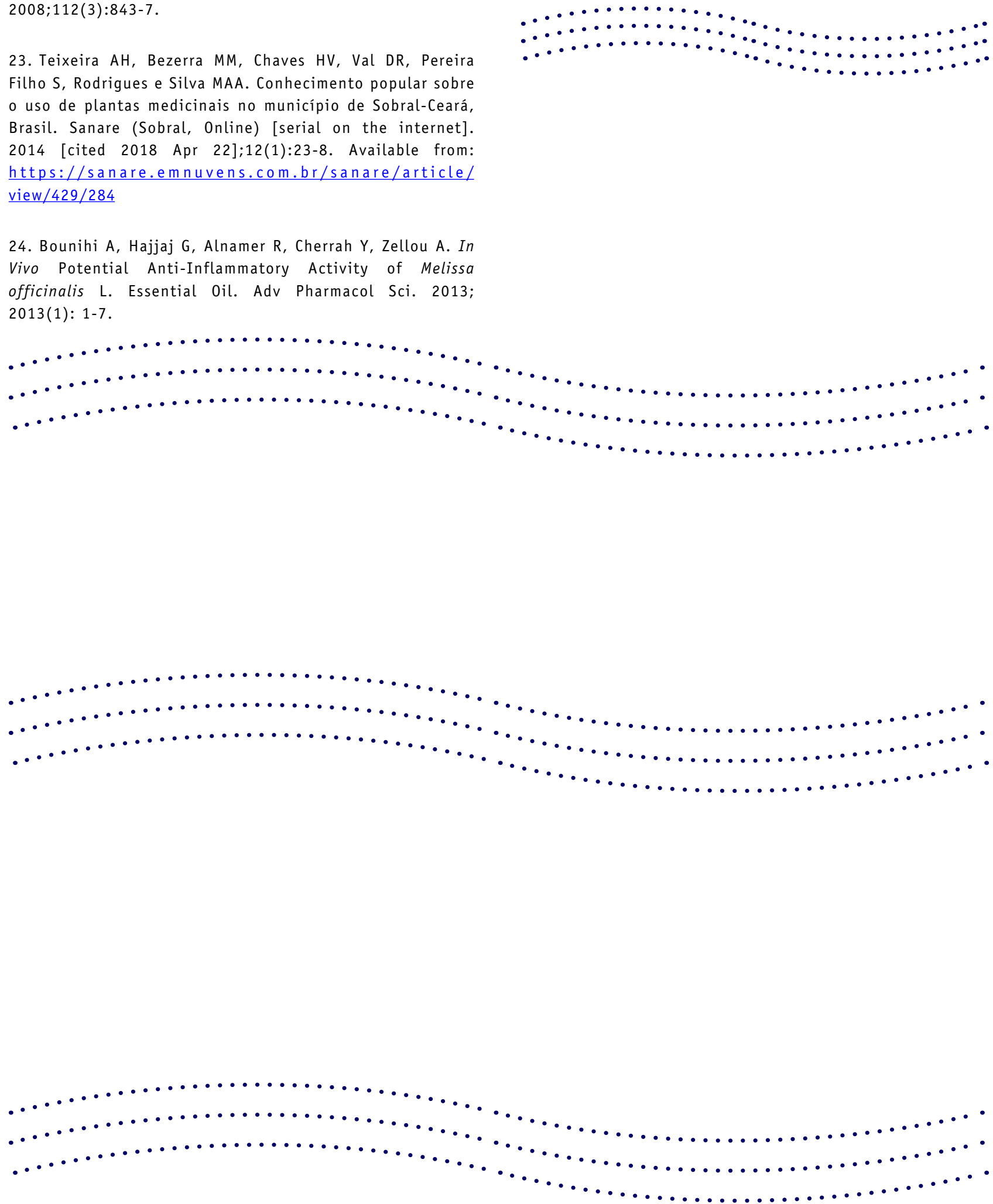